SHEP-07-11

FNT/T 2007-04

October 23, 2018

\title{
Vector-Boson Production of Light Higgs Pairs in 2-Higgs Doublet Models
}

\author{
M. Moretti \\ Dipartimento di Fisica, Università di Ferrara and \\ INFN - Sezione di Ferrara, Via Paradiso 12, 44100 Ferrara, Italy \\ S. Moretti \\ School of Physics 85 Astronomy, University of Southampton, \\ Highfield, Southampton SO17 1BJ, UK, and \\ Laboratoire de Physique Théorique, Université Paris-Sud, F-91405 Orsay Cedex, France \\ F. Piccinini \\ INFN - Sezione di Pavia, Dipartimento di Fisica Nucleare e Teorica, \\ Via Bassi 6, 27100 Pavia, Italy \\ R. Pittau 1 \\ Dipartimento di Fisica Teorica, Università di Torino and \\ INFN - Sezione di Torino, Via Giuria 1, 10125 Torino, Italy, and \\ Departamento de Física Teórica y del Cosmos, \\ Centro Andaluz de Física de Partículas Elementales (CAFPE), \\ Universidad de Granada, E-180\%1 Granada, Spain \\ J. Rathsman \\ High Energy Physics, Uppsala University, Box 535, 75121 Uppsala, Sweden
}

\begin{abstract}
At the Large Hadron Collider, we prove the feasibility to detect pair production of the lightest CP-even Higgs boson $h$ of Type II 2-Higgs Doublet Models through $q q^{\left({ }^{\prime}\right)} \rightarrow$ $q q^{\left({ }^{\prime}\right)} h h$ (vector-boson fusion). We also show that, through the $h h \rightarrow 4 b$ decay channel in presence of heavy-flavour tagging, further exploiting forward/backward jet sampling, one has direct access to the $\lambda_{H h h}$ triple Higgs coupling - which constrains the form of the Higgs potential.
\end{abstract}

\footnotetext{
${ }^{1}$ Present address: Institute of Nuclear Physics, NCSR "DEMOKRITOS", 15310, Athens, Greece.
} 


\section{Introduction}

If only a light Higgs boson (with mass $M_{h} \lesssim 140 \mathrm{GeV}$ ) is found at the Large Hadron Collider (LHC), it may be difficult to tell whether it belongs to the Standard Model (SM) or indeed a model with an enlarged Higgs sector. For example, in the case of a CP-conserving Type II 2-Higgs Doublet Model (2HDM) [1]-[4]2, possibly in presence of minimal Supersymmetry (SUSY) - the combination of the two yielding the so-called Minimal Supersymmetric Standard Model (MSSM) - this happens in the so-called 'decoupling region', when $M_{H}, M_{A}, M_{H^{ \pm}} \gg M_{h}$, for suitable choices of the other MSSM and 2HDM parameters, where - for the same mass - the $h$ couplings to ordinary matter in the SM are the same as in both the 2HDM and MSSM. Even in these conditions, however, it has been proved that one could possibly establish the presence of an extended Higgs sector by determining the size of the trilinear Higgs self-coupling $\lambda_{h h h}[5]$.

If the extended model is not in a decoupling condition, then it is generally possible to establish the presence of additional Higgs signals, $H, A$ and/or $H^{ \pm}$[6, 7]. However, even when this is the case, it may be difficult to distinguish, e.g., between a generic Type II 2HDM and the MSSM (unless, of course, one also detects the SUSY partners of ordinary matter and Higgs bosons). In fact, despite there exist well establish spectra among the four different masses in the MSSM (for fixed, say, $M_{h}$ and $\tan \beta$, the ratio of the vacuum expectation values of the two Higgs doublets in either model), it may well be possible that the additional 2HDM parameters arrange themselves to produce an identical mass pattern. However, such a degeneracy between the two models would not typically persist if one were able to also measure certain Higgs couplings, chiefly those among the Higgs bosons themselves (involving two or more such particles). In fact, while the measurement of only two among the four Higgs boson masses $\left(M_{h}, M_{H}, M_{A}\right.$ and $M_{H^{ \pm}}$) - or, alternatively, one such masses and $\tan \beta$ - would fix (at tree-level) all Higgs masses and couplings in the MSSM, this is no longer true in a generic Type II 2HDM [1], because of the freedom in selecting the free additional parameters. For example, the general CP-conserving Type II 2HDM that we are going to consider can be specified uniquely by seven parameters: $M_{h}, M_{H}, M_{A}, M_{H^{ \pm}}, \beta, \alpha$ (the mixing angle between the two CP-even neutral Higgs states) and $\lambda_{5}$ (see eq. (2) later on). It may then happen that the first six of these are measured and found to agree with the MSSM pattern, but one would still need to measure $\lambda_{5}$ to verify that it is the Higgs sector of the MSSM that is present. One way to do so would be by measuring trilinear Higgs self-couplings, such as $\lambda_{h h h}$ and $\lambda_{H h h}$. Alternatively, the measurement of the latter two couplings would constitute a test of the MSSM relations if one knew $M_{h}$ and $\tan \beta$ but not $\alpha$.

In this paper, we make the assumption that only one parameter is known, $M_{h}$, as may well happen at the LHC after only a $h$ resonance is detected. We further imply that all

\footnotetext{
${ }^{2}$ Of the initial eight degrees of freedom pertaining to the two complex Higgs doublets, only five survive as real particles upon Electro-Weak Symmetry Breaking (EWSB), labelled as $h, H, A$ (the first two are CP-even or 'scalars' (with $M_{h}<M_{H}$ ) whereas the third is CP-odd or 'pseudoscalar') and $H^{ \pm}$, as three degrees of freedom are absorbed into the definition of the longitudinal polarisation for the gauge bosons $Z$ and $W^{ \pm}$, upon their mass generation after EWSB.
} 
(potential) SUSY states are much heavier than ordinary particles (with the possible exception of the lightest SUSY particle, see footnote 4), thus effectively a decoupled MSSM setup. Under these circumstances, we then ask ourselves the following question. While trying to establish the presence of additional (single) heavy Higgs signals, which would then unmistakably distinguish between the SM and a scenario with an extended Higgs sector, would it also be possible to gather information on Higgs self-couplings from signatures involving two light Higgs bosons, hence by studying channels involving $h$ pair production, thereby possibly also distinguish between, e.g., a generic Type II 2HDM and the MSSM?

It is the purpose of this paper to show that this is the case, so long that enough luminosity can be accumulated at the LHC, also in view of the Super-LHC (SLHC) option [8]. We will illustrate how we have come to this conclusion, i.e., after investigating the process [9]

$$
q q^{\left({ }^{\prime}\right)} \rightarrow q q^{\left({ }^{\prime}\right)} h h(\text { vector }- \text { boson fusion })
$$

with $q^{\left({ }^{\prime}\right)}$ referring to any possible (anti)quark flavour combinations. The relevant Feynman diagrams corresponding to process (11) in both the MSSM and 2HDM considered here can be found in Fig. 1. In our selection analysis, we will resort to the extraction of two $h \rightarrow b \bar{b}$ resonances, in presence of the following signature:

- 'four b-quark jets and two forward/backward-jets'.

This signature was already considered in Ref. [5] in the SM context (from which we will import some of the results).

Our paper is organised as follows. In the next section, we outline the computational procedure. Sect. 3 presents our numerical results and discusses these in various subsections. Sect. 4 contains our conclusions.

\section{Calculation}

We have assumed $\sqrt{s}=14 \mathrm{TeV}$ for the LHC energy throughout. Our numerical results are obtained by setting the renormalisation and factorisation scales to $2 M_{h}$ for the signal while for the QCD background we have used the average jet transverse momentum $\left(p_{T}^{2}=\sum_{1}^{n} p_{T j}^{2} / n\right)$. Both Higgs processes and noise were estimated by using the Parton Distribution Function (PDF) set MRST99(COR01) [18]. While the background calculations were based on exact treelevel Matrix Elements (MEs) using the ALPGEN program [19], all signal rates were obtained

\footnotetext{
${ }^{3}$ The gluon-gluon production mode [10] was considered in Refs. [11] and [12] (see also [13]), and later on [14, 15, where - despite significant kinematic differences exist between signal and QCD noise - it was eventually shown that the extraction of the $g g \rightarrow h h \rightarrow b \bar{b} b \bar{b}$ signal is essentially impossible at the (S)LHC because of the overwhelming QCD noise, both reducible and irreducible. Recently, encouraging results on the cross-section for multi-Higgs boson production in the gluon-gluon production mode has been obtained in models beyond the SM and MSSM [16. The possibility of using Higgs boson pair production more generally to access trilinear Higgs couplings has also been studied on the level of total cross-sections in [17.
} 
through the same level of accuracy via programs based on the HELAS subroutines [20] - for the computation of the MEs - and VEGAS [21] or Metropolis [22] - for the multi-dimensional integrations over the phase space. As for numerical input values of SM parameters, we adopted the ALPGEN defaults.

Concerning the MSSM setup, the two independent tree-level parameters that we adopt are $M_{A}$ and $\tan \beta$. Through higher orders, we have considered the so called 'Maximal Mixing' scenario $\left(X_{t}=A_{t}-\mu / \tan \beta=\sqrt{6} M_{\mathrm{SUSY}}\right)$ [23], wherein we have chosen for the relevant SUSY input parameters: $\mu=200 \mathrm{GeV}, A_{b}=0$, with $M_{\mathrm{SUSY}}=5 \mathrm{TeV}$, the latter - as already intimated - implying a sufficiently heavy scale for all sparticle masses, so that these are not accessible at the LHC and no significant interplay between the SUSY and Higgs sectors of the model can take place 4 . Masses and couplings within the MSSM have been obtained by using the HDECAY program [24].

Before giving the details of the 2HDM setup we are using, let us recall the most general CP-conserving $2 \mathrm{HDM}$ scalar potential which is symmetric under $\Phi_{1(2)} \rightarrow-\Phi_{1(2)}$ up to softly breaking dimension-2 terms (thereby allowing for loop-induced flavour changing neutral currents) [1],

$$
\begin{aligned}
V= & m_{11}^{2} \Phi_{1}^{\dagger} \Phi_{1}+m_{22}^{2} \Phi_{2}^{\dagger} \Phi_{2}-\left\{m_{12}^{2} \Phi_{1}^{\dagger} \Phi_{2}+\text { h.c. }\right\}+\frac{1}{2} \lambda_{1}\left(\Phi_{1}^{\dagger} \Phi_{1}\right)^{2}+\frac{1}{2} \lambda_{2}\left(\Phi_{2}^{\dagger} \Phi_{2}\right)^{2}+ \\
& +\lambda_{3}\left(\Phi_{1}^{\dagger} \Phi_{1}\right)\left(\Phi_{2}^{\dagger} \Phi_{2}\right)+\lambda_{4}\left(\Phi_{1}^{\dagger} \Phi_{2}\right)\left(\Phi_{2}^{\dagger} \Phi_{1}\right)+\left\{\frac{1}{2} \lambda_{5}\left(\Phi_{1}^{\dagger} \Phi_{2}\right)^{2}+\text { h.c. }\right\}
\end{aligned}
$$

In the following, the parameters $m_{11}, m_{22}, m_{12}, \lambda_{1}, \lambda_{2}, \lambda_{3}$ and $\lambda_{4}$ are replaced by $v, M_{h}, M_{H}$, $M_{A}, M_{H^{ \pm}}, \beta$ and $\alpha$ (with $v$ fixed). Hence, as intimated already, the CP-conserving $2 \mathrm{HDM}$ potential is parameterised by seven free parameters. Notice that from the scalar potential all the different Higgs couplings needed for our study can easily be obtained. (See [2, 3] for a complete compilation of couplings in a general CP-conserving 2HDM.)

In our $2 \mathrm{HDM}$, we will fix $M_{h}$ and $M_{H}$ to values similar to the ones found in the MSSM scenario we are considering, by adopting three different setups:

1. $M_{h}=115 \mathrm{GeV}, M_{H}=300 \mathrm{GeV}$,

2. $M_{h}=115 \mathrm{GeV}, M_{H}=500 \mathrm{GeV}$,

3. $M_{h}=115 \mathrm{GeV}, M_{H}=700 \mathrm{GeV}$.

We always scan over the remaining parameters in the ranges

$$
\begin{gathered}
-\pi / 2<\alpha<\pi / 2 \\
-4 \pi<\lambda_{5}<4 \pi \\
0<\tan \beta<50
\end{gathered}
$$

\footnotetext{
${ }^{4}$ The only possible exception in this mass hierarchy would be the Lightest Supersymmetric Particle (LSP), whose mass may well be smaller than the lightest Higgs mass values that we will be considering. However, we have verified that invisible $h$ decays (including the one into two LSPs) have negligible decay rates.
} 


$$
\begin{gathered}
100 \mathrm{GeV}<M_{A}<1000 \mathrm{GeV}, \\
100 \mathrm{GeV}<M_{H^{ \pm}}<1000 \mathrm{GeV} .
\end{gathered}
$$

In order to accept a point from the scan we also check that the following conditions are fulfilled: the potential is bounded from below, the $\lambda_{i}$ fulfill the tree-level unitarity constraints of [25] and yield a contribution to $|\Delta \rho|<10^{-3}$. In short the unitarity constraints amounts to putting limits on the eigen values of the $S$ matrices for scattering various combinations of Higgs and electroweak gauge bosons. We have followed the normal procedure [1] of requiring the $J=0$ partial waves $\left(a_{0}\right)$ of the different scattering processes to fulfill $\left|\operatorname{Re}\left(\mathrm{a}_{0}\right)\right|<1 / 2$, which corresponds to applying the condition that the eigenvalues $5 \Lambda_{Y \sigma \pm}^{Z_{2}}$ of the scattering matrices (or more precisely $16 \pi S$ ) fulfill $\left|\Lambda_{Y \sigma \pm}^{Z_{2}}\right|<8 \pi$ [26]. In other words we allow parameter space points all the way up to the tree-level unitarity constraint $\left|\operatorname{Re}\left(\mathrm{a}_{0}\right)\right|<1 / 2$. In order to investigate the sensitivity to this upper limit we will also report results as a function of the value of the maximal eigenvalue, $\Lambda_{\max }$. The spectrum of masses, couplings and decay rates in our $2 \mathrm{HDM}$ is the same as in Ref. [27], obtained by using a modification of HDECAY [24] (consistent with a similar manipulation of the program used in Ref. [28]). For each accepted point in the scan the partial decay rates for the different Higgs bosons are then calculated using HDECAY and also taking possible additional partial widths of the $H$ into account.

While the parameter dependence of the MSSM Higgs sector renders the computation of the tree-level MSSM cross-sections rather straightforward (as the latter depends on two parameters only, $M_{A}$ and $\tan \beta$ ), the task becomes much more time-consuming in the context of the $2 \mathrm{HDM}$. In order to calculate the cross-sections in this scenario, they are schematically written as a combination of couplings and kinematic factors in the following way:

$$
\sigma_{\mathrm{tot}}=\int\left|\sum_{i=1}^{5} g_{i} M_{i}\right|^{2} d \operatorname{LIPS}=\sum_{i=1}^{5} \sum_{j=i}^{5} g_{i} g_{j} \sigma_{i j}
$$

where all the explicit dependence on $\alpha, \beta, \lambda_{H h h}$ and $\lambda_{h h h}$ is contained in the couplings $g_{i}$ : $g_{1}=\sin ^{2}(\beta-\alpha), g_{2}=\cos ^{2}(\beta-\alpha), g_{3}=\cos (\beta-\alpha) \lambda_{H h h}, g_{4}=\sin (\beta-\alpha) \lambda_{h h h}$, and $g_{5}=1$, whereas the dependence on masses and other couplings is in the factors

$$
\sigma_{i j}=\frac{1}{1+\delta_{i j}} \int\left(M_{i}^{\dagger} M_{j}+M_{j}^{\dagger} M_{i}\right) d \mathrm{LIPS} .
$$

Note that the sum over subamplitudes $M_{i}$ also contains all interference terms and that colour factors etc. are included properly 6 The $\sigma_{i j}$ are then calculated numerically for fixed masses. We can then get the cross-section in an arbitrary parameter space point by multiplying the kinematic factors with the appropriate couplings. However, there is a slight complication since the kinematic factor for the $H \rightarrow h h$ contribution depends on the width $\Gamma_{H}$ if there is a schannel resonance and the width in turn depends on the couplings. In this case the kinematic factor scales as $1 / \Gamma_{H}$ which is accounted for by assuming a fixed value for the width when the

\footnotetext{
${ }^{5}$ Here, $Z_{2}$ refers to the $Z_{2}$ symmetry, $Y$ is the hypercharge, and $\vec{\sigma}$ is the total weak isospin

${ }^{6}$ We have carefully verified the integrity of our procedure.
} 
kinematic factor is calculated and then rescaling the result with the true width when calculating the contribution to the cross-section. Another complication is the dependence of the kinematic factors on the Higgs masses, $M_{A}$ and $M_{H^{ \pm}}$. The contributions of main interest, which contain the $\lambda_{H h h}$ and $\lambda_{h h h}$ couplings, only depend on these masses indirectly through the unitarity constraints. At the same time there are other contributions to the cross-section which depend explicitly on these masses. However, these contributions are very small in the parts of parameter space of interest and can thus be safely neglected.

\section{Results}

In our investigation of the emerging hadronic final state, we will assume that $b$-quark jets are distinguishable from light-quark and gluon ones and neglect considering $b$-jet charge determination. Finite calorimeter resolution has been emulated through a Gaussian smearing in transverse momentum, $p_{T}$, with $\left(\sigma\left(p_{T}\right) / p_{T}\right)^{2}=\left(0.60 / \sqrt{p_{T}}\right)^{2}+(0.04)^{2}$, for all jets. The corresponding missing transverse momentum, $p_{T}^{\text {miss }}$, was reconstructed from the vector sum of the visible momenta after resolution smearing. Finally, in our parton level analysis, we have identified jets with the partons from which they originate and applied all cuts directly to the latter, since parton shower and hadronisation effects were not included in our study.

\subsection{Inclusive Signal Results}

In this section, after a preliminary analysis of the Higgs mass and coupling spectra in the MSSM and a general Type II 2HDM, we will start our numerical analysis by investigating the model parameter dependence of the Higgs pair production process in (1) at fully inclusive level, in presence of the decay of the latter into two $b \bar{b}$ pairs, with the integration over the phase space being performed with no kinematical restrictions. This will be followed by an analysis of the production and decay process pertaining to the Higgs signal of interest at fully differential level, in presence of detector acceptance cuts and kinematical selection constraints. Finally, we will compare the yield of the signal to that of the corresponding background and perform a dedicated signal-to-background study including an optimisation of the cuts in order to enhance the overall significance. We will treat the MSSM and 2HDM in two separate subsections.

\subsubsection{MSSM}

As representative of the low and high $\tan \beta$ regime, we will use in the remainder the values of 3 and 40. We have instead treated $M_{A}$ as a continuous parameter, varying between 100 and 700 $\mathrm{GeV}$ or sd7. Before proceeding with the numerical analysis of the signal, it is worthwhile to investigate both the Higgs mass and coupling dependence in the MSSM with respect to the two input parameters $M_{A}$ and $\tan \beta$. This is done in Figs. 2 and 3, respectively. In the latter, we

\footnotetext{
${ }^{7}$ Values of $M_{A}$ below $90 \mathrm{GeV}$ or so are actually excluded by LEP for the lower $\tan \beta$ value: see [29].
} 
study the case of (CP-even) MSSM Higgs boson couplings to gauge bosons (denoted by $G_{h V V}$ and $G_{H V V}$ ), wherein $V$ refers to either a $W^{ \pm}$or a $Z$. In the same figure, the symbol $\phi$ refers to the SM Higgs boson, with mass identical to that of the lightest MSSM Higgs state $\left(M_{\phi}=M_{h}\right)$. While the pattern of masses has been well established in past literature, it is interesting to notice here that the product of the MSSM couplings entering process (1) is always smaller than in the SM case. However, in the MSSM, resonance enhancements can occur (such as in $H \rightarrow h h$ ), so that the actual MSSM production rates can in some cases overcome the corresponding SM ones (for $M_{\phi}=M_{h}$ ).

Fig. 4 presents the fully inclusive MSSM cross-section for the process of interest, as defined in (11), times (effectively) $\mathrm{BR}(h h \rightarrow b \bar{b} b \bar{b})$. The shape of the curves is mainly dictated by the interplay between phase space (see Fig. 2) and coupling (see Fig. 31) effects, with the exception of the region $M_{A} \gtrsim 220 \mathrm{GeV}$ and $\tan \beta=3$, where the onset of the $H \rightarrow h$ resonance is clearly visible. Cross-sections are generally sizable, particularly at low $\tan \beta$. The displayed rates however coincide to the ideal situation in which all final state jets are detected with unit efficiency and the detector coverage extend to their entire phase space, so that they only serve as a guidance in rating the phenomenological relevance of the process discussed.

A more realistic analysis is in order, which we have performed as follows. The four $b$-jets emerging from the decay of the $h h$ pair are accepted according to the following criteria:

$$
p_{T}^{b}>30 \mathrm{GeV}, \quad\left|\eta^{b}\right|<2.5, \quad \Delta R_{b b}>0.7
$$

in transverse momentum, pseudorapidity and cone separation, respectively. Their tagging efficiency is taken as $\epsilon_{b}=50 \%$ for each $b$ satisfying these requirements, $\epsilon_{b}=0$ otherwise 8 . In addition, to enforce the reconstruction of the two Higgs bosons, we require all such $b$ 's in the event to be tagged and that at least one out of the three possible double pairings of $b$-jets satisfies the following mass preselection:

$$
\left(m_{b_{1}, b_{2}}-M_{h}\right)^{2}+\left(m_{b_{3}, b_{4}}-M_{h}\right)^{2}<2 \sigma_{m}^{2}
$$

where $\sigma_{m}=0.12 M_{h}$. We further exploit 'forward/backward-jet' tagging, by imposing that the non- $b$-jets satisfy the additional cuts

$$
p_{T}^{\text {fwd } / \text { bwd }}>20 \mathrm{GeV}, \quad 2.5<\eta^{\text {fwd }}<5, \quad-2.5>\eta^{\text {bwd }}>-5 .
$$

Tab. 11 shows the rates of the signal after the implementation of the constraints in eqs. (15)-(7) (hereafter, referred to as 'acceptance and preselection cuts' or 'primary cuts'). While our process does yield non-negligible rates after the latter, it turns out that it is of no phenomenological relevance, even assuming very high luminosity. Firstly, in view of the fact that $b$-tagging efficiencies are not taken into account in this table: for the ' $4 b$-jet' tagging option, one should multiply the numbers in Tab. 1 by $\epsilon_{b}^{4}$, that is, 1/16. (Alternative approaches requiring a lesser

\footnotetext{
${ }^{8}$ Here and in the remainder, the label $b$ refers to jets that are $b$-tagged while $j$ to any jet (even those originating from $b$-quarks) which is not.
} 


\begin{tabular}{|c|c|c|c|}
\hline \multicolumn{4}{|c|}{$\tan \beta=3$} \\
\hline$M_{A}(\mathrm{GeV})$ & $M_{h}(\mathrm{GeV})$ & $\sigma\left(q q^{\left({ }^{(}\right)} \rightarrow q q^{\left({ }^{\prime}\right)} h h\right)[\mathrm{fb}]$ & $\sigma$ (background) [fb] \\
\hline 160 & 108 & 0.19 & 218 \\
200 & 112 & 0.23 & 232 \\
240 & 114 & 0.46 & 229 \\
\hline \hline \multicolumn{4}{|c|}{$\tan \beta=40$} \\
\hline$M_{A}(\mathrm{GeV})$ & $M_{h}(\mathrm{GeV})$ & $\sigma\left(q q^{\left({ }^{(}\right)} \rightarrow q q^{\left({ }^{\prime}\right)} h h\right)[\mathrm{fb}]$ & $\sigma$ (background) [fb] \\
\hline 160 & 129 & 0.26 & 224 \\
200 & 129 & 0.20 & 224 \\
240 & 129 & 0.17 & 224 \\
\hline
\end{tabular}

Table 1: Cross-sections for Higgs pair production via vector-boson fusion, process (11), after Higgs boson decays (relevant BRs are all included) and the acceptance and preselection cuts defined in (5)-(7), for two choices of $\tan \beta$ and a selection of $M_{A}$ values, assuming the MSSM in Maximal Mixing configuration (the corresponding values of $M_{h}$ are also indicated in brackets). No $b$-tagging efficiencies are included here.

number of $b$-jets to be tagged as such were not successful either.) Secondly, the background rates, after the same cuts in eqs. (5)-(7), are always overwhelming the signal, despite our efforts in further optimising the cuts. For this reason, rather than dwelling upon the latter now, we postpone their discussion to the next subsection and simply conclude here that our channel is altogether inaccessible at both the LHC and SLHC in the context of the MSSM.

\subsubsection{HDM}

As already alluded to, the parameter space of the general CP-conserving Type II 2HDM we are considering is quite large as it depends on seven unknown parameters. In order to get a feel for the dependence of the signal cross-section for the process $q q^{\left({ }^{\prime}\right)} \rightarrow q q^{\left({ }^{\prime}\right)} h h \rightarrow q q^{\left({ }^{\prime}\right)} b \bar{b} b \bar{b}$ we therefore present in Figs. 5 through 7 the results of our three selected scenarios, wherein we scan the allowed parameter space over 10000 randomly chosen points. (Note that similarly to the MSSM case we have included the $\mathrm{BR}(h h \rightarrow b \bar{b} b \bar{b})$ but not any $4 b$-jet tagging efficiency.)

Comparing with the cross-sections in the MSSM the main differences are due to the following:

- the triple Higgs couplings $9 \lambda_{H h h}$ and $\lambda_{h h h}$ are not related to the gauge couplings;

- the different parameters can vary independently of each other.

Conversely, the kinematic factors in the two models will be the same for a given set of masses and widths of the different Higgs bosons. Therefore, in those cases, many features of the signal,

\footnotetext{
${ }^{9}$ We use the same definitions of these couplings as in 2 .
} 


\begin{tabular}{|c|l|l|l|l|}
\hline$M_{H}(\mathrm{GeV})$ & \multicolumn{4}{|c|}{$\sigma\left(q q^{\left({ }^{\prime}\right)} \rightarrow q q^{\left({ }^{\prime}\right)} h h\right)[\mathrm{fb}]$ with different cuts } \\
\hline & inclusive & primary & optimal & optimal, $H \rightarrow h h$ \\
\hline \hline 300 & 1453 & 71.9 & 31.2 & 25.8 \\
\hline 500 & 396 & 25.3 & 11.4 & 7.7 \\
\hline 700 & 80 & 7.1 & 3.3 & 2.0 \\
\hline
\end{tabular}

Table 2: The maximal cross-sections in the $2 \mathrm{HDM}$ under consideration for $M_{h}=115 \mathrm{GeV}$, and $M_{H}=300,500$ and $700 \mathrm{GeV}$, respectively, with the following different cuts: inclusive, with primary cuts in eqs. (5)-(7), and with optimised cuts of eq. (8) in the latter case also when only considering the $H \rightarrow h h$ resonant contribution.

such as the differential distributions, will be similar to those of the MSSM even though the normalisation can be completely different. In fact, comparing Fig. 4 with 5 through 7 we see that in the more general 2HDM the cross-sections can be more than two orders of magnitude larger than in the MSSM thus rendering a much larger potential for a detectable signal (as it will be discussed below). To be more quantitative on this we give in Tab. 2 the maximal inclusive cross-sections obtained in the scans for $M_{h}=115 \mathrm{GeV}$ and $M_{H}=300,500$ and $700 \mathrm{GeV}$.

In order to study the potential signal in more detail we first of all apply the same primary cuts as in the case of the MSSM, those listed in eqs. (5)-(7). The resulting cross-sections are given Tab. 2. Comparing with the cross-section without the primary cuts we see that the reduction is substantial, but even so the signal cross-section can still be more than two orders of magnitude larger than in the MSSM scenario considered in subsection 3.1.1 and it is comparable to the background (see Tab. 1, specifically for low $\tan \beta$, where the $M_{h}$ values in the two models are very similar).

\subsection{Signal-to-Background Differential Analysis}

In this section, we will continue the discussion of our numerical analyses limitedly to the Type II $2 \mathrm{HDM}$ considered so far. In order to enhance the statistical significance $S / \sqrt{B}$ we studied several differential distributions for signals and background with the event selection of eqs. (51)(7), with the aim of introducing optimised cuts, allowing at the same time to keep the signal event numbers at a reasonable level. To begin with, for simplicity, we have limited ourselves to use the contribution from the $H \rightarrow h h$ resonance to the signal for $M_{H}=300 \mathrm{GeV}$ in a scenario where the cross-section is close to maximal, with $\cos (\beta-\alpha)=1, \lambda_{H h h}=1000 \mathrm{GeV}$ and $\Gamma_{H}=30 \mathrm{GeV}$, when comparing with the background.

The most sensitive distributions, able to discriminate between the signal and background, turn out to be the minimum transverse momentum of the forward/backward jets and the nextto-minimum invariant mass of the $b \bar{b}$ pairs, which we show in Fig. 8. (Although to a some more 
limited extent, also the minimum $b \bar{b}$ invariant mass is useful.) Before selecting a specific set up, we performed also a systematic analysis of the significance for different combination of cuts $\left(30 \mathrm{GeV} \leq m_{b b}^{\min } \leq m_{b b}^{\text {next-to-min }} \leq 100 \mathrm{GeV}, 20 \mathrm{GeV} \leq p_{\mathrm{T}}^{\mathrm{fwd}} \leq 60 \mathrm{GeV}\right)$. The best optimised cuts, on top of the basic ones of eqs. (5)-(7), that we found are 10 :

$$
p_{T}^{\text {fwd/bwd }}>40 \mathrm{GeV}, \quad m_{b b}^{\min }>40 \mathrm{GeV}, \quad m_{b b}^{\text {next-to-min }}>80 \mathrm{GeV} .
$$

We show in Fig. 9 the $4 b$ invariant mass distribution for three signals $\left(M_{H}=300,500\right.$ and $700 \mathrm{GeV}$ with the widths $\Gamma_{H}=30,50$ and $200 \mathrm{GeV}$, respectively) and the background after the optimised cuts of eq. (8) have also been imposed. For each of the three signals shown in the figure we have used the parameter space point which gives the maximal signal cross-section from the resonant $H \rightarrow h h$ contribution when restricting the width $\Gamma_{H}$ to be less than 30, 50 and $200 \mathrm{GeV}$, respectively. In this context we note that there are two effects which mainly determine the width of the signal distribution. On the one hand, the smearing of momenta we use gives a contribution to the measurable width of about $30 \mathrm{GeV}$. On the other hand, one of course has the intrinsic width of the $H$.

Taking suitable mass windows around the peaks for the different Higgs mass values illustrated in Fig. 9, we obtain the maximal signal cross-sections, event numbers and statistical significances quoted in Tab. 3. In order to calculate the signal cross-sections in the respective windows for different parameter space points, taking the actual width of the $H$ into account, we rescaled the contribution from the $H \rightarrow h h$ resonance with a factor $c_{M_{H}} \equiv$ $\left(\arctan \left[2\left(M_{H}-m_{L}\right) / \Gamma_{i}\right]+\arctan \left[2\left(m_{U}-M_{H}\right) / \Gamma_{i}\right]\right)$ where $m_{L}$ and $m_{U}$ are the lower and upper limits of the signal window, $\Gamma_{i}$ is the width of the signal distribution in parameter space point $i$ estimated from $\Gamma_{i}=\sqrt{\Gamma_{H_{i}}^{2}+\Gamma_{4 \mathrm{~b}}^{2}}$ with $\Gamma_{4 \mathrm{~b}}=30 \mathrm{GeV}$ being the width of the $m_{4 b}$-distributions from finite detector resolution. (Notice then that $c_{M_{H}}$ is a normalisation determined from scenarios with $\Gamma_{H_{i}}=30,50$ and $200 \mathrm{GeV}$ for the different $H$ masses.) Thus we approximate the cross-section in the $m_{4 b}$ window as $\sigma_{\text {peak }}=c_{M_{H}} \sigma_{H \rightarrow h h}$. As a further requirement we also imposed that at least $50 \%$ of the signal cross-section after the optimal cuts comes from the $H \rightarrow h h$ resonance such that the would-be-signal would not be obscured by other non-resonant contributions.

The distributions of the signal cross-sections obtained in this way are given in Fig. 10, For illustration, the $5 \sigma$ limits at LHC, assuming an integrated luminosity of $300 \mathrm{fb}^{-1}, \sigma_{\text {peak }}>2.7$ $(2.3-3.3) 11$, 1.5 (1.3-1.8) and $0.8(0.7-1.0) \mathrm{fb}$, for $M_{H}=300,500$ and $700 \mathrm{GeV}$ respectively, are

\footnotetext{
${ }^{10}$ Note that the efficiency of these is rather insensitive to the actual Higgs mass values, so that we have used the same set for any choice of the latter.

${ }^{11}$ The ranges given within parenthesis in this paragraph have been obtained by varying the factorisation/renormalisation scale for the background by a factor of two around the default value, which makes the corresponding cross-section decrease by $30 \%$ or increase by $50 \%$ respectively. The reason for this is that, being essentially a six-jet cross-section, the background rate is proportional to $\alpha_{s}^{6}$ and it is therefore quite sensitive to the renormalisation scale. We also note that our default scale $\left(p_{T}^{2}=\sum_{1}^{n} p_{T j}^{2} / n\right)$ has conservatively been chosen to be small so, if anything, our estimate of the final signal-to-background rates should be regarded as conservative. In a real experiment one should of course attempt to use the sidebands for background normalisation.
} 


\begin{tabular}{|l|l|l|l|l|l|}
\hline$m_{4 b}$ window & $B$ events & $\sigma_{\text {peak }}^{\max }[\mathrm{fb}]$ & $S$ events & $S / \sqrt{B} @$ LHC & $S / \sqrt{B} @$ SLHC \\
\hline $280-340(\mathrm{GeV})$ & 102 & 15.1 & 283 & 28 & 89 \\
\hline $460-540(\mathrm{GeV})$ & 30 & 3.8 & 71 & 13 & 41 \\
\hline $660-740(\mathrm{GeV})$ & 8 & 0.35 & 6.6 & 2.3 & 7.4 \\
\hline
\end{tabular}

Table 3: Number of events and significances for $M_{h}=115 \mathrm{GeV}$ and $M_{H}=300,500,700 \mathrm{GeV}$ in the respective best case scenarios, for a $4 b$-tagging efficiency of $(50 \%)^{4}$ and after the optimised cuts of eq. (8). The assumed integrated luminosity at LHC and SLHC are $300 \mathrm{fb}^{-1}$ and $3000 \mathrm{fb}^{-1}$, respectively.

also illustrated and the fractions of parameter space points which gives cross-sections larger then this are 27 (24-30), 8 (5-12) and $0 \%$, respectively. The corresponding numbers for the SLHC with $3000 \mathrm{fb}^{-1}$ are 43 (41-45), 31 (28-33) and 2 (0-2)\%, respectively. Thus even at the SLHC we find no scope of observing a $M_{H}=700 \mathrm{GeV}$ resonance in the channel under investigation.

Finally we have also investigated the effects of restricting the allowed parameter space from tree-level unitarity by putting harder constraints on the maximal eigenvalue of the scattering matrices, $\Lambda_{\max }$. For this purpose, Fig. 11] shows the signal cross-sections obtained in the scan as a function of $\Lambda_{\max }$. From the figure it is clear that the results (at least for $M_{H}=300$ and 500 $\mathrm{GeV}$ ) are not sensitive to the precise value used for applying the unitarity constraint. On the other hand, applying a much harder constraint of the order $\Lambda_{\max } \lesssim 4(12)$ (instead of $\Lambda_{\max }<8 \pi$ ) essentially leads to that the sensitivity for detection at the LHC is more or less washed out for $M_{H}=300(500) \mathrm{GeV}$. The same also holds at the SLHC assuming an integrated luminosity of $3000 \mathrm{fb}^{-1}$.

\section{Conclusions}

We would like to conclude our paper by stating that, at both the LHC and SLHC, there exists a great potential to extract a $H \rightarrow h h \rightarrow 4 b$ resonance when $M_{h}$ is constrained in the vicinity of $115 \mathrm{GeV}$. This is a crucial result if one recalls that the detection of a sole Higgs resonance and consequent extraction of an $M_{h}$ value may not point unambiguously to the underlying model of EWSB, not even in presence of further measurements of the heavier Higgs masses, $M_{H}, M_{A}$ and/or $M_{H^{ \pm}}$.

For example, the $2 \mathrm{HDM}$ considered here may be realised in a configuration wherein all visible Higgs masses are degenerate with those of the MSSM. Under these circumstances, we have proved that

- it is not possible to extract an $H \rightarrow h h \rightarrow 4 b$ resonance from vector-boson fusion in the $\operatorname{MSSM}$ (not even if $M_{H}$ is known) whilst 
- the opposite case is true in a substantial fraction of the parameter space of our 2HDM (even if $M_{H}$ is not known), thereby enabling one to possibly measure the triple-Higgs coupling $\lambda_{H h h}$.

The latter is a Lagrangian term, which is different between these two models even when their patterns of Higgs masses and couplings to SM objects are the same, that would give a unique insight into the underlying EWSB mechanism.

To be more specific our results show that in the most favourable scenario with $M_{H}=300$ GeV up to 27 (43) \% of the parameter space would give a $5 \sigma$ signal at the (S)LHC assuming an integrated luminosity of $300(3000) \mathrm{fb}^{-1}$ when using the standard tree-level unitarity requirement on the $J=0$ partial waves, $\operatorname{Re}\left(a_{0}\right)<1 / 2$. These results are not sensitive to the precise value used for applying the unitarity constraint, albeit for very strong constraints the sensitivity for detecting the signal goes away. In the case of $M_{H}=500 \mathrm{GeV}$ the fraction of parameter space probed is smaller with up to 8 (31) \% giving a $5 \sigma$ signal, whereas for $M_{H}=700 \mathrm{GeV}$ there is essentially no sensitivity at all.

Despite we lack a full Monte Carlo simulation we believe to have incorporated the most critical aspects of the latter so that we do not expect more realistic studies (including parton shower, hadronisation, heavy hadron decays and detector effects) to affect too strongly our conclusions.

Finally, we are currently pursuing other work along the directions outlined here, covering the case of lightest (neutral) Higgs boson pair production in the case of Higgs-strahlung and in association with heavy quarks [30].

\section{Acknowledgments}

We are all grateful to the (formerly) CERN Theory Division for hospitality when this work was started. Several discussions with M.L. Mangano are acknowledged. SM and FP thank JR for his kind hospitality in Uppsala in September 2006. FP thanks SM for his stay in Southampton in March and June 2007. RP acknowledges the financial support of the MIUR under contract 2006020509_004 and of the RTN European Programme MRTN-CT-2006-035505 (HEPTOOLS, Tools and Precision Calculations for Physics Discoveries at Colliders). SM acknowledges the latter too for partial funding. RP's research was partially supported by the ToK Program "ALGOTOOLS" (MTKD-CT-2004-014319). FP thanks the CERN Theory Unit for partial support.

\section{References}

[1] J.F. Gunion, H.E. Haber, G.L. Kane and S. Dawson, "The Higgs Hunter Guide” (AddisonWesley, Reading MA, 1990), Erratum, hep-ph/9302272. 
[2] J.F. Gunion and H.E. Haber, Phys. Rev. D67 (2003) 075019.

[3] F. Boudjema and A.V. Semenov, Phys. Rev. D66 (2002) 095007.

[4] A. Djouadi, arXiv:hep-ph/0503172; arXiv:hep-ph/0503173.

[5] M. Moretti, S. Moretti, F. Piccinini, R. Pittau and A.D. Polosa, JHEP 02 (2005) 04; hep-ph/0411039.

[6] ATLAS collaboration, 'ATLAS Technical proposal', CERN/LHCC/94-43, LHCC/P2, 1994; 'ATLAS Detector and Physics Performance Technical Design Report', ATLAS TDR 14, CERN/LHCC 99-14, 1999.

[7] CMS collaboration, 'CMS Technical Proposal', CERN/LHCC/94-38, LHCC/P1, 1994; 'CMS Physics Technical Design Report, Volume I: Detector Performance and Software' CMS TDR 8.1, CERN/LHCC 2006-001, 2006.

[8] F. Gianotti, M.L. Mangano and T. Virdee (conveners), hep-ph/0204087.

[9] W.-Y. Keung, Mod. Phys. Lett. A10 (1987) 765; O.J.P. Éboli, G.C. Marques, S.F. Novaes and A.A. Natale, Phys. Lett. B197 (1987) 269; D.A. Dicus, K.J. Kallianpur and S.S.D. Willenbrock, Phys. Lett. B200 (1988) 187; K.J. Kallianpur, Phys. Lett. B215 (1988) 392; A. Abbasabadi, W.W. Repko, D.A. Dicus and R. Vega, Phys. Rev. D38 (1988) 2770, Phys. Lett. B213 (1988) 386; A. Dobrovolskaya and V. Novikov, Z. Phys. C52 (1991) 427.

[10] E.W.N. Glover and J.J. van der Bij, Nucl. Phys. B309 (1988) 282; D.A. Dicus, C. Kao and S.S.D. Willenbrock, Phys. Lett. B203 (1988) 457; G. Jikia, Nucl. Phys. B412 (1994) 57; T. Plehn, M. Spira and P.M. Zerwas, Nucl. Phys. B479 (1996) 46, Erratum, ibidem B531 (1998) 655.

[11] R. Lafaye, D.J. Miller, M. Muhlleitner and S. Moretti, hep-ph/0002238.

[12] A. Djouadi, R. Kinnunen, E. Richter-Was, H.U. Martyn et al., in hep-ph/0002258.

[13] E. Richter-Was et al., Int. J. Mod Phys. A13 (1998) 1371; E. Richter-Was and D. Froidevaux, Z. Phys. C76 (1997) 665; J. Dai, J.F. Gunion and R. Vega, Phys. Lett. B371 (1996) 71, ibidem, B378 (1996) 801.

[14] U. Baur, T. Plehn and D. Rainwater, Phys. Rev. D68 (2003) 033001.

[15] S. Balatenychev et al., in hep-ph/0203056.

[16] T. Binoth, S. Karg, N. Kauer and R. Ruckl, Phys. Rev. D74 (2006) 113008.

[17] A. Djouadi, W. Kilian, M. Muhlleitner and P. M. Zerwas, Eur. Phys. J. C 10 (1999) 45. 
[18] http://durpdg.dur.ac.uk/hepdata/pdf.html.

[19] M.L. Mangano, M. Moretti, F. Piccinini, R. Pittau and A.D. Polosa, JHEP 07 (2003) 001.

[20] H. Murayama, I. Watanabe and K. Hagiwara, KEK Report 91-11, January 1992.

[21] G.P. Lepage, J. Comp. Phys. 27 (1978) 192, preprint CLNS-80/447, March 1980.

[22] H. Kharraziha and S. Moretti, Comp. Phys. Comm. 127 (2000) 242, Erratum, ibidem 134 (2001) 136 .

[23] M. Carena, P.H. Chankowski, S. Pokorski and C.E.M. Wagner, Phys. Lett. B441 (1998) 205.

[24] A. Djouadi, J. Kalinowski and M. Spira, Comp. Phys. Comm. 108 (1998) 56.

[25] A.G. Akeroyd, A. Arhrib and E.M. Naimi, Phys. Lett. B490 (2000) 119.

[26] I. F. Ginzburg and I. P. Ivanov, Phys. Rev. D 72 (2005) 115010.

[27] S. Moretti and J. Rathsman, Eur. Phys. J. C33 (2004) 41.

[28] S. Moretti and W.J. Stirling, Phys. Lett. B347 (1995) 291, Erratum, ibidem B366 (1996) 451.

[29] See: http://lephiggs.web.cern.ch/LEPHIGGS/papers/.

[30] M. Moretti, S. Moretti, F. Piccinini, R. Pittau and J. Rathsman, in preparation. 


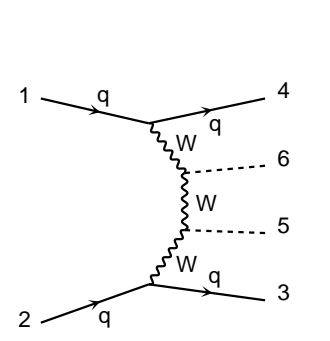

Diagrams by FEYNMANDRAW
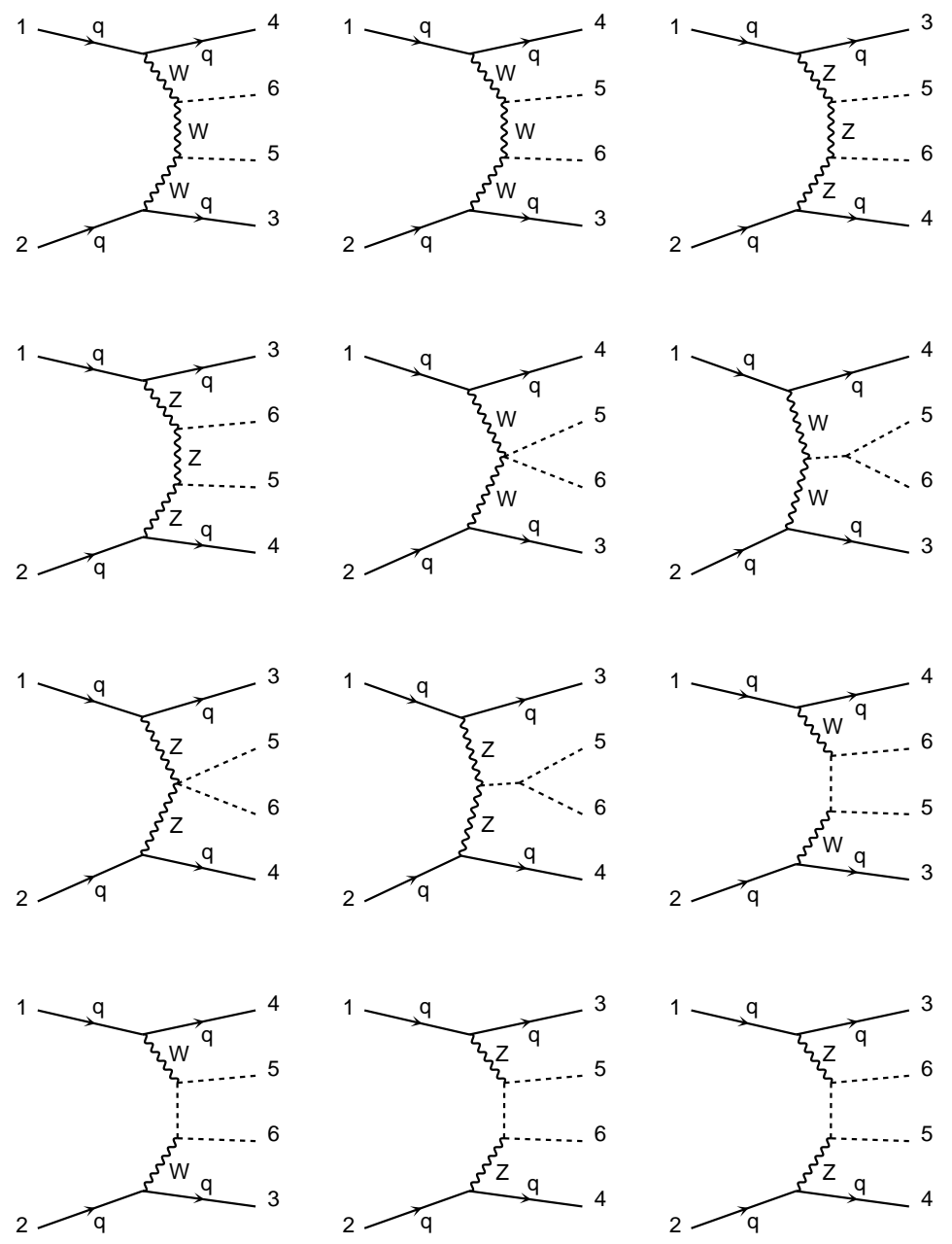

Figure 1: Feynman diagrams for $q_{1} q_{2} \rightarrow q_{3} q_{4} h_{5} h_{6}$. Depending on the (anti)quark flavour combination, the $W^{ \pm}$- and $Z$-mediated graphs may not interfere. Besides, for final state (anti)quarks of different flavours, only half of the diagrams survive. 


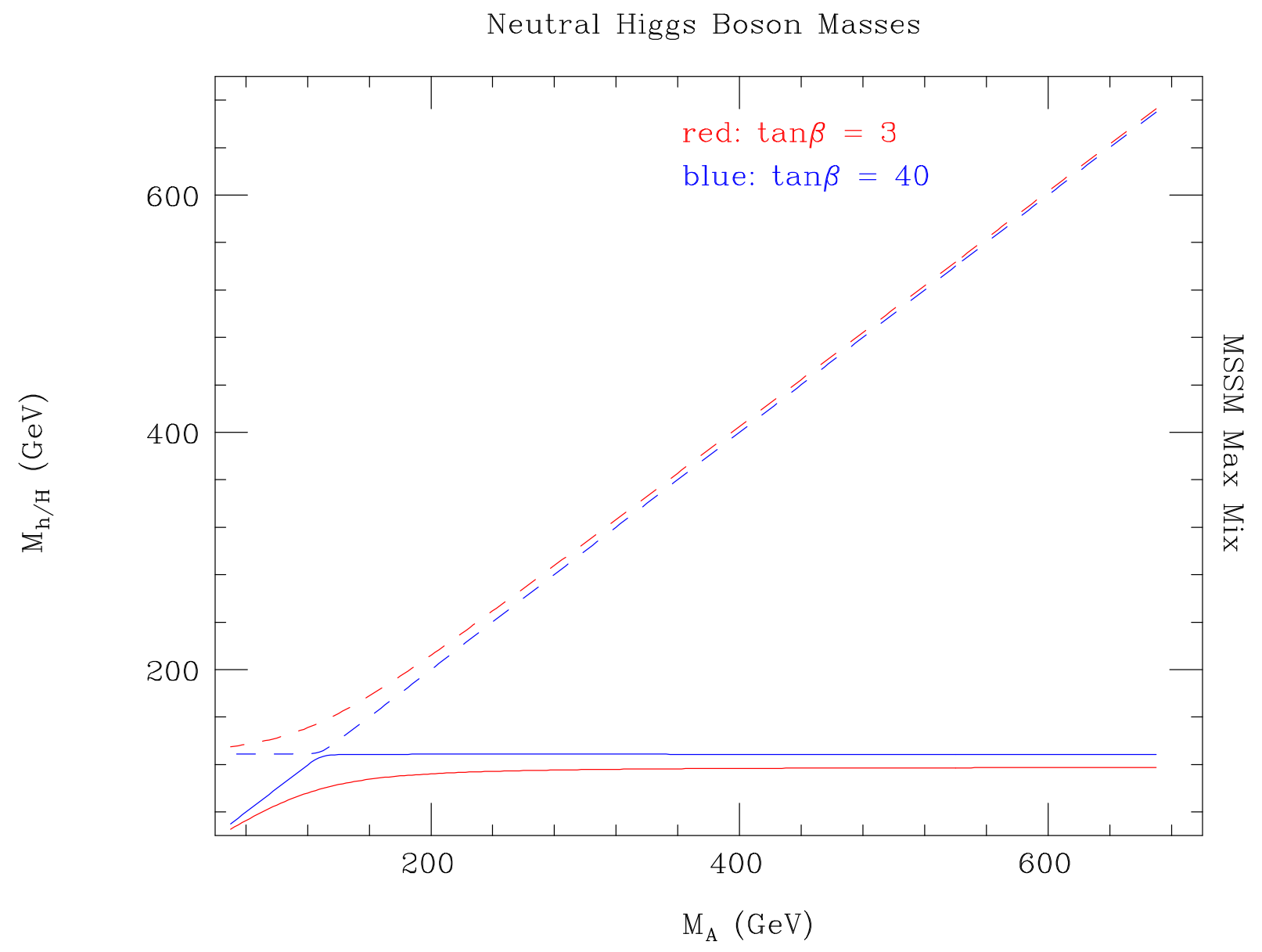

Figure 2: The masses of the neutral CP-even Higgs bosons as a function of the CP-odd one, for two choices of $\tan \beta$, assuming the MSSM in Maximal Mixing configuration. 


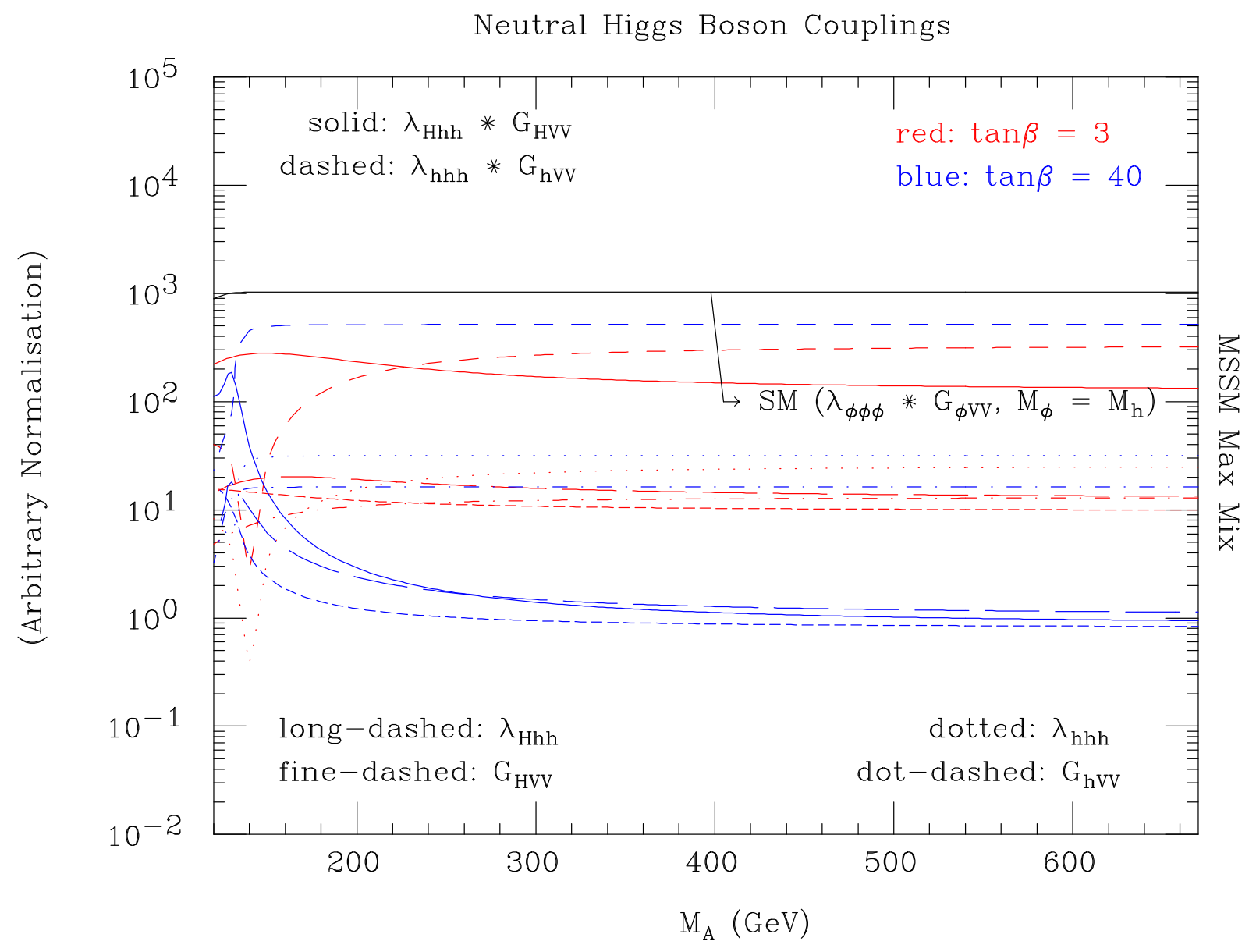

Figure 3: The relevant couplings of the neutral CP-even Higgs bosons entering the production process in (11) as a function of the CP-odd Higgs boson mass, for two choices of tan $\beta$, assuming the MSSM in Maximal Mixing configuration. 


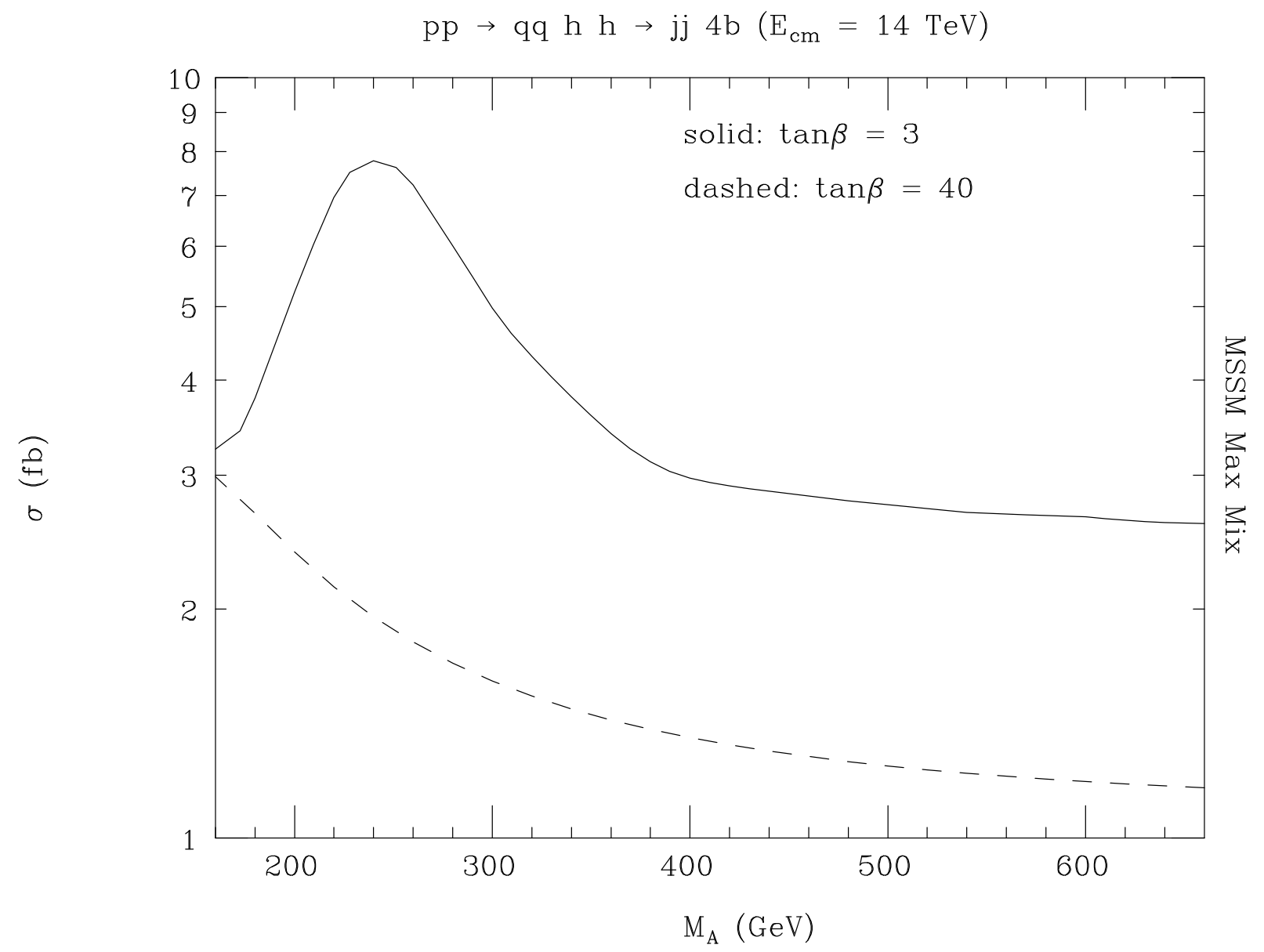

Figure 4: The inclusive cross-sections (as defined in the text) for vector-boson fusion in (1), followed by $h h \rightarrow b \bar{b} b \bar{b}$ decays, as a function of the CP-odd Higgs boson mass, for two choices of $\tan \beta$, assuming the MSSM in Maximal Mixing configuration. 

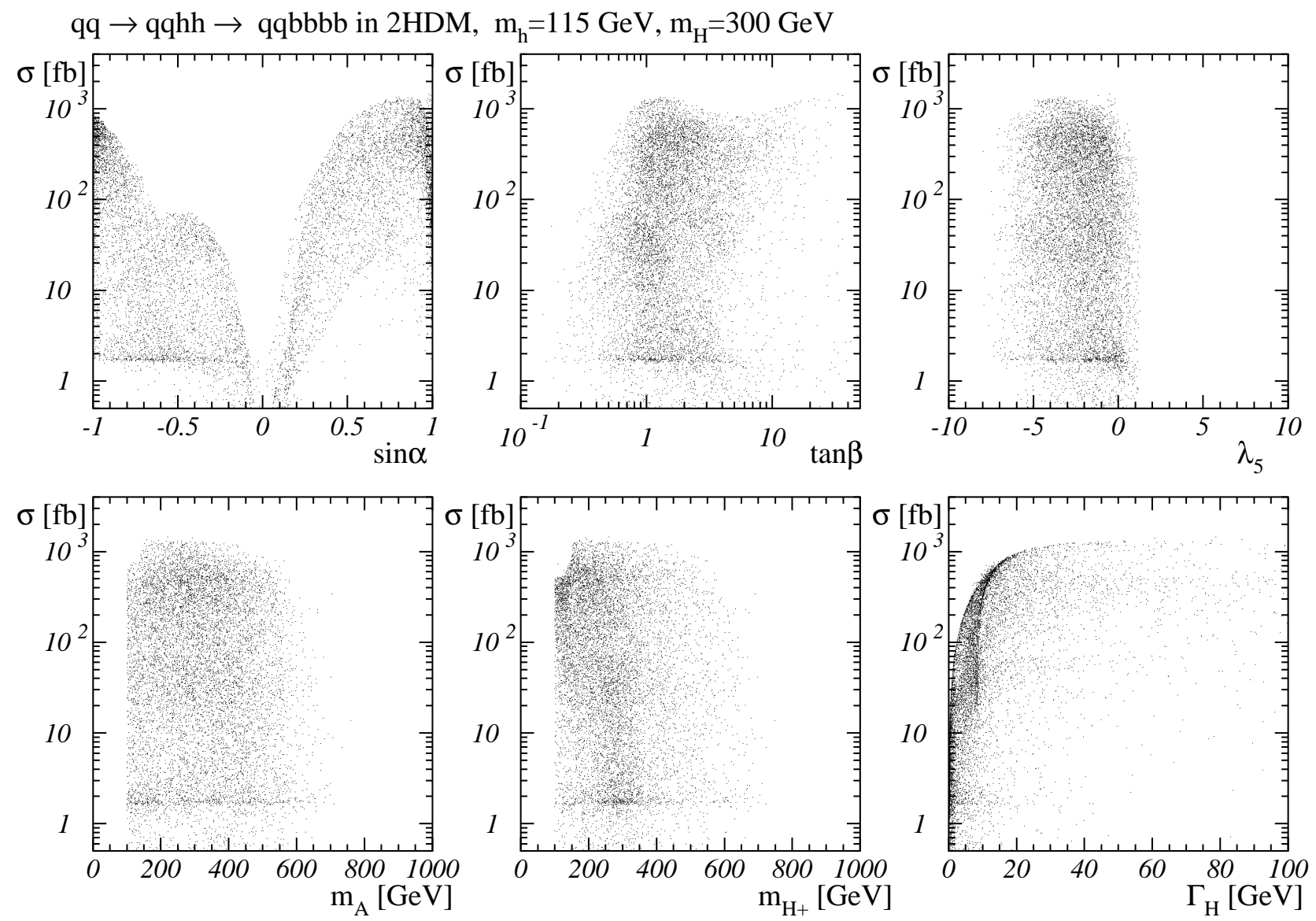

Figure 5: The dependence of the inclusive cross-section $q q^{\left({ }^{(}\right)} \rightarrow q q^{\left({ }^{\prime}\right)} h h \rightarrow q q^{\left({ }^{(}\right)} b \bar{b} b \bar{b}$ in the 2HDM under consideration on the different parameters when scanning over 10000 parameter space points for $M_{h}=115 \mathrm{GeV}$ and $M_{H}=300 \mathrm{GeV}$. (Note that $M_{A}$ and $M_{H^{ \pm}}$are free parameters.) 

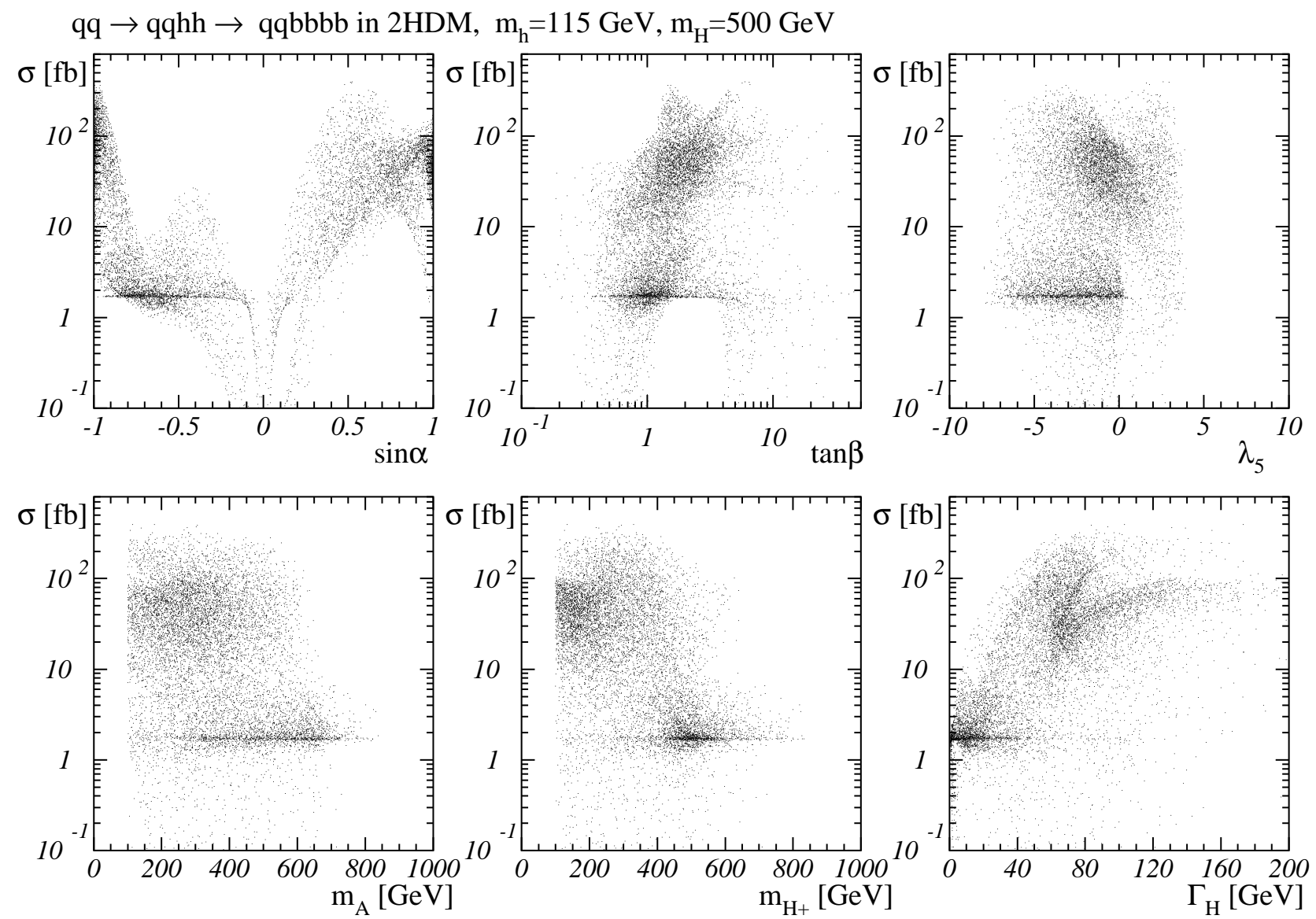

Figure 6: The dependence of the inclusive cross-section $q q^{\left({ }^{(}\right)} \rightarrow q q^{\left({ }^{\prime}\right)} h h \rightarrow q q^{\left({ }^{(}\right)} b \bar{b} b \bar{b}$ in the 2HDM under consideration on the different parameters when scanning over 10000 parameter space points for $M_{h}=115 \mathrm{GeV}$ and $M_{H}=500 \mathrm{GeV}$. (Note that $M_{A}$ and $M_{H^{ \pm}}$are free parameters.) 

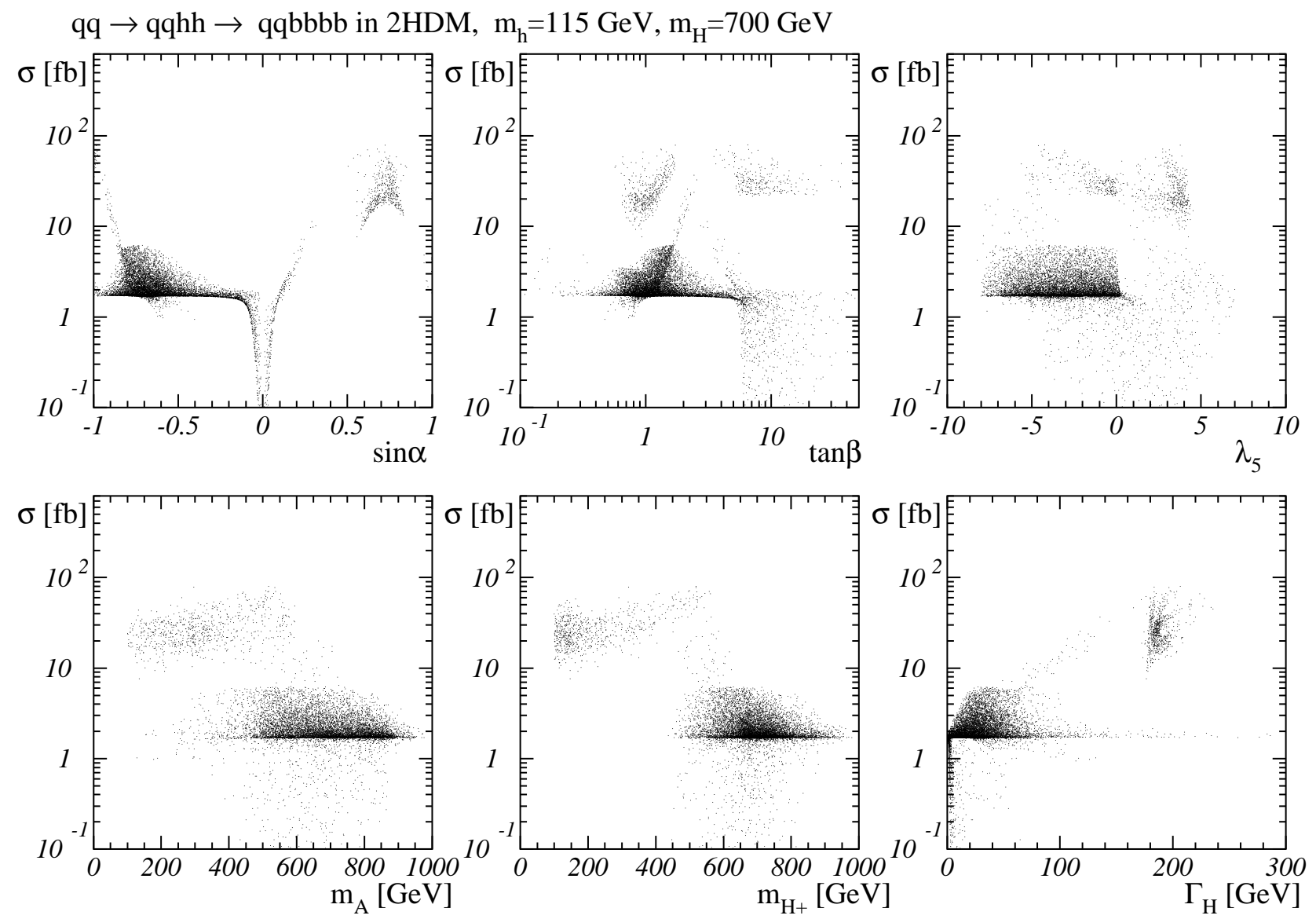

Figure 7: The dependence of the inclusive cross-section $q q^{\left({ }^{(}\right)} \rightarrow q q^{\left({ }^{\prime}\right)} h h \rightarrow q q^{\left({ }^{(}\right)} b \bar{b} b \bar{b}$ in the 2HDM under consideration on the different parameters when scanning over 10000 parameter space points for $M_{h}=115 \mathrm{GeV}$ and $M_{H}=700 \mathrm{GeV}$. (Note that $M_{A}$ and $M_{H^{ \pm}}$are free parameters.) 

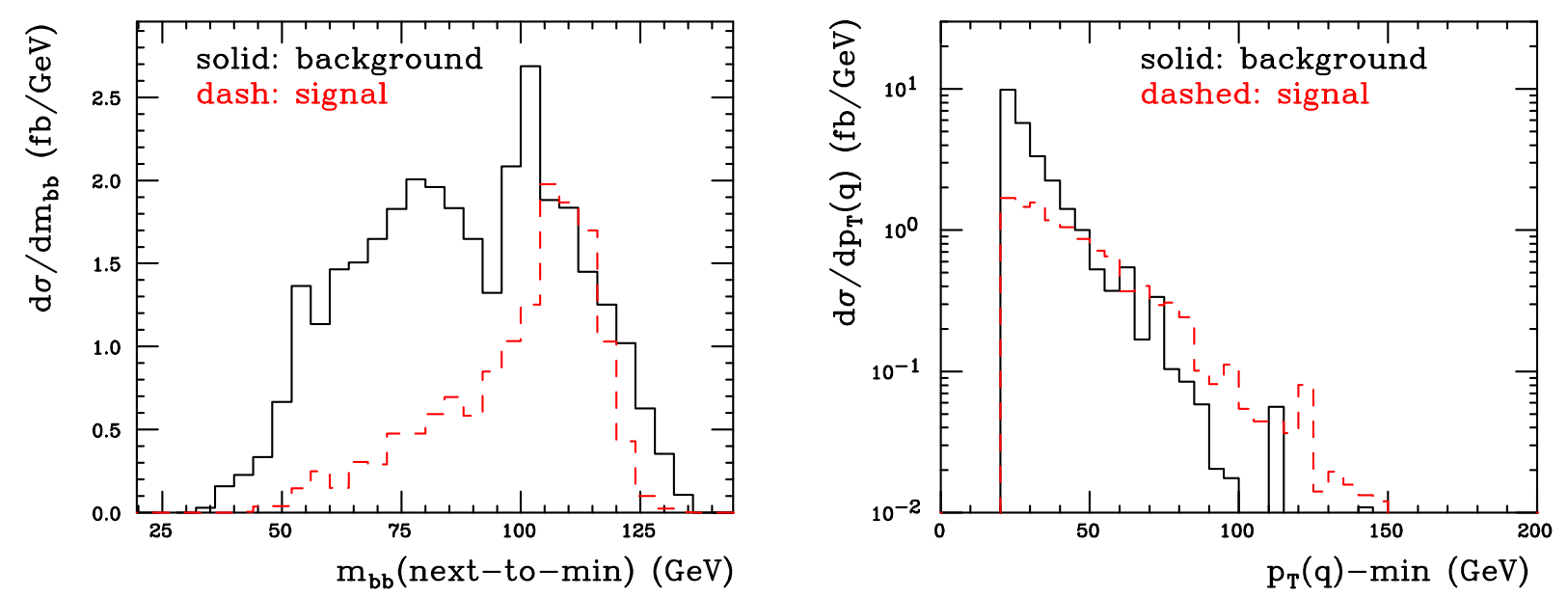

Figure 8: The distribution of the next-to-minimum $b \bar{b}$ invariant mass (left) and of the minimum tagging jet transverse momentum (right) for the signal (cross section for $q q^{\left({ }^{\prime}\right)} \rightarrow q q^{\left({ }^{\prime}\right)} h h \rightarrow$ $q q^{\left({ }^{\prime}\right)} b \bar{b} b \bar{b}$ in a close to best-case scenario for $M_{H}=300 \mathrm{GeV}$ ) and the background. The basic cuts of eqs. (15)-(17) are imposed. 


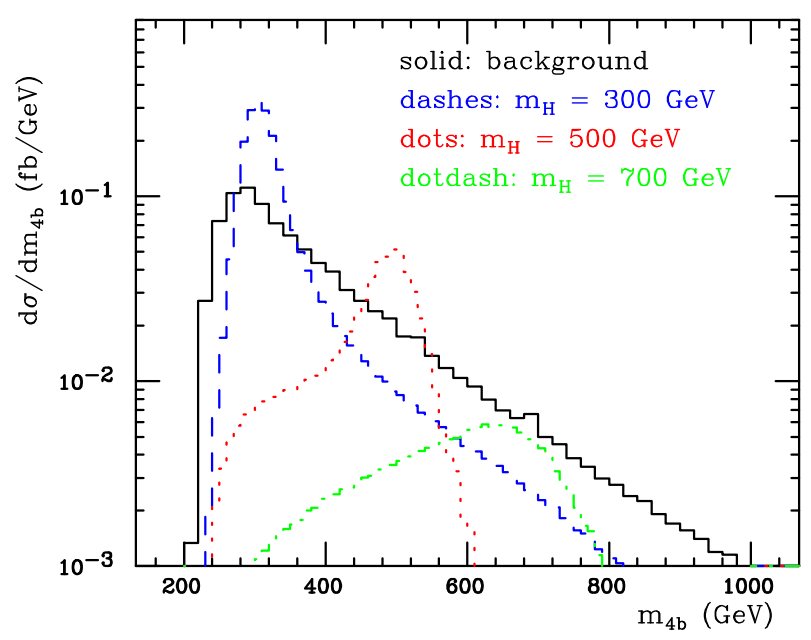

Figure 9: The differential cross-section $d \sigma\left(q q^{\left({ }^{\prime}\right)} \rightarrow q q^{\left({ }^{\prime}\right)} h h \rightarrow q q^{\left({ }^{\prime}\right)} b \bar{b} b \bar{b}\right) / d m_{4 b}$ in the best case scenarios for $M_{H}=300,500$ and $700 \mathrm{GeV}$ obtained when scanning over the available parameter space restricting the width $\Gamma_{H}$ to be less than 30, 50 and $200 \mathrm{GeV}$, respectively. When calculating the signal distributions the actual widths have been assumed to be $\Gamma_{H}=30,50$ and $200 \mathrm{GeV}$, respectively. 

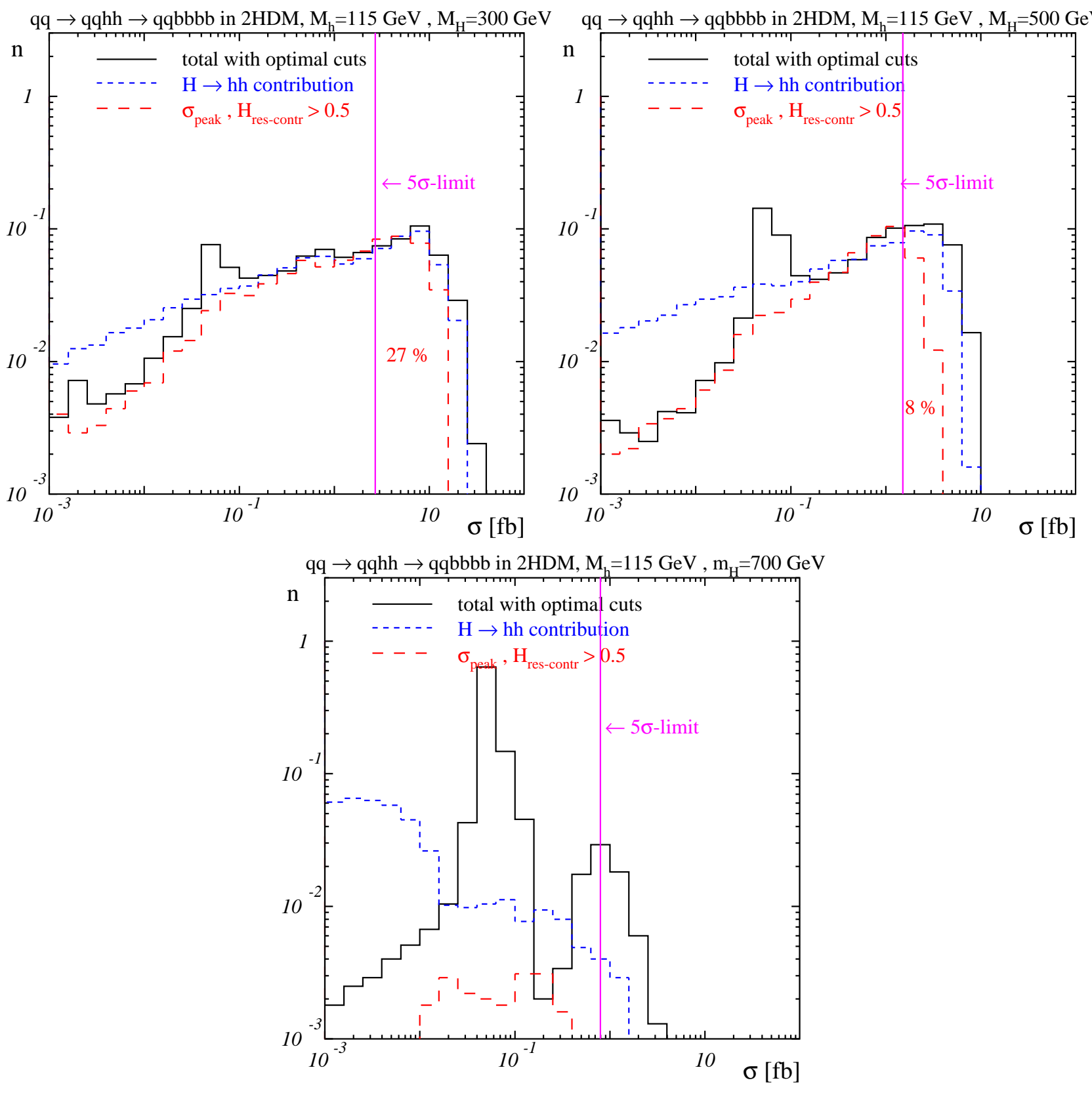

Figure 10: Distributions of the resulting cross-sections $q q^{\left({ }^{\prime}\right)} \rightarrow q q^{\left({ }^{\prime}\right)} h h \rightarrow q q^{\left({ }^{\prime}\right)} b \bar{b} b \bar{b}$ in the 2HDM under consideration using the optimal cuts obtained in a scan over 10000 parameter space points (the area is normalised to 1 for the cross-section with optimal cuts) for three different sets of Higgs boson masses as indicated in the respective plots. The solid line shows the results with optimal cuts, the dashed line shows the resonant contribution from the $H \rightarrow h h$ processes and the long dashed line shows the resonant contribution in the respective signal windows requiring that at least $50 \%$ of the cross-section comes from the $H \rightarrow h h$ resonance. The vertical line corresponds to the $5 \sigma$-limit at LHC assuming $300 \mathrm{fb}^{-1}$ and the integral of the curves to the right of it gives the percentage of parameter space points where the resonant cross-section is larger than this. 

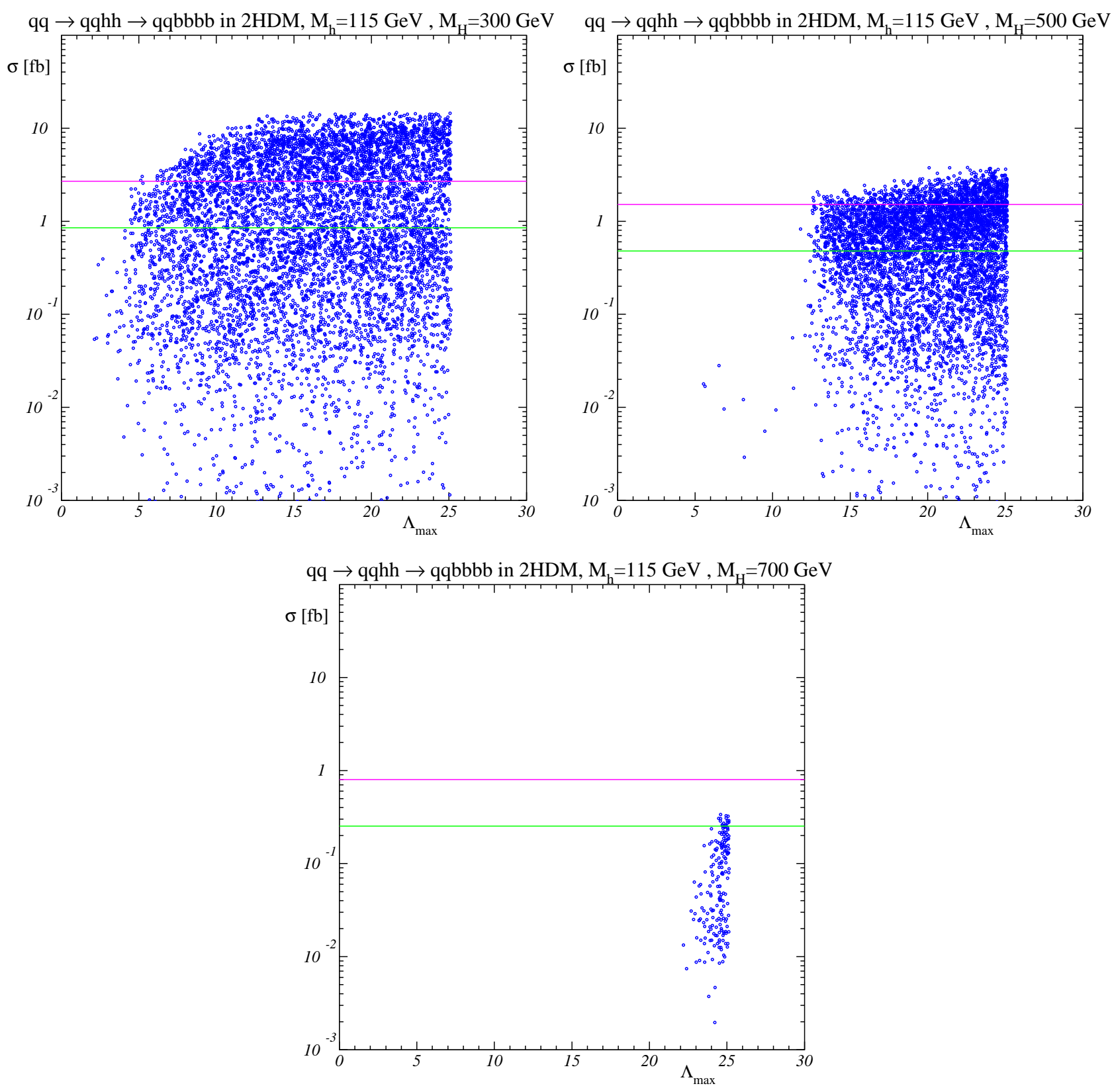

Figure 11: The distributions in resulting signal cross-sections $q q^{\left({ }^{\prime}\right)} \rightarrow q q^{\left({ }^{\prime}\right)} h h \rightarrow q q^{\left({ }^{\prime}\right)} b \bar{b} b \bar{b}$ in the $2 \mathrm{HDM}$ under consideration using the optimal cuts obtained in a scan over 10000 parameter space points as a function of the maximal eigenvalue $\Lambda_{\max }$ of the scattering matrix for three different sets of Higgs boson masses as indicated in the respective plots. The upper (lower) horizontal line corresponds to the $5 \sigma$-limit at (S)LHC assuming $300 \mathrm{fb}^{-1}\left(3000 \mathrm{fb}^{-1}\right)$. 